\title{
Technical and economic efficiency of proactive diagnostic systems for power transformers
}

\author{
A.N. Alyunov ${ }^{1}$, O.S. Vyatkina ${ }^{1}, A . V$. Udaratin $^{1}$ and D.A. Zaripova ${ }^{2}$ \\ ${ }^{1}$ Vologda State University, Vologda, Russia \\ ${ }^{2}$ Kazan State Power Engineering University, Kazan, Russia
}

\begin{abstract}
The expediency of using systems of proactive diagnostics of power transformers is substantiated. The diagnostic system expands the possibilities of analyzing the transformer condition in operating mode. It allows one to minimize the expected damage from interruptions in power supply and equipment failure. The results can be used in world practice during construction of various control systems to identify developing transformer defects caused by windings deformations.
\end{abstract}

\section{Introduction}

The strategy of international scientific and technological development comprises transition to advanced digital, intelligent manufacturing technologies, robotic systems, new materials and design methods, creation of systems for processing large volumes of data, machine learning and artificial intelligence.

Under the current conditions, it is possible to bring the properties of protections installed on transformers closer to ideal. It is achieved by implementation of proactive actions of relay protection by identifying potential damage at early stage of its development. This significantly reduces the risk of sudden failures in transformers, and, therefore, the risk of sudden disturbances in power system.

Internal short-circuit in transformers of $110-500 \mathrm{kV}$ are caused, first of all, by damage of on-load tapchangers, high-voltage bushings and windings. The most severe consequences occur during development of the following defects [1]:

- Reduction of electric strength of oil channel of high-voltage sealed bushings due to deposition of sediment on the inner surface of porcelain and on the surface of internal insulation;

- Reduction of electric strength of paper-oil insulation of high-voltage unsealed bushings due to wetting and pollution;

- Pollution, moisture and wear of insulation of transformer windings;

- Burnout of winding insulation and turns of windings due to prolonged non-shutdown of through short-circuit current on the low voltage side of transformer;

- Errors during installation, operation and repairs.

The causes, behavior, and consequences of damage to transformer windings are well understood, however, traditional protection can detect damage when $65-70 \%$ of the winding turns are closed, which is clearly not enough. Moreover, various damages, including deformation of windings, may remain unnoticed, which leads to the further progress of emergency situations and conversion of winding faults to multiphase short circuits. The disadvantage of the existing methods is the large turn-off time of winding circuits and uneven sensitivity to symmetric and asymmetric short circuits.

It should be noted that most of these defects could be timely detected using methods and means of technical diagnostics $[2,3]$.

There are methods for diagnostics of power transformers according to the experience of idling and short circuit, requiring disconnection of transformer from the network. There are systems for transformer monitoring that calculate an integrated coefficient of current technical condition of transformer. Registration of currents and voltages of the primary and secondary windings of transformer in such modules is used to calculate only short circuit resistance. These approaches do not consider other parameters (short circuit losses, current and idle losses), so they have a decreased accuracy of damage diagnosis [4-6].

The system of diagnostics of transformers and other equipment should have full information, technical, regulatory support, as well as a decision-making strategy on the possibility and expediency of its further operation or the need for repair.

The main guidance documents regulate the list of transformer tests during commissioning and operation, the maximum permissible values of the controlled parameters and deadline monitoring. In recent times, there appeared a need for a significant enhancement of the controlled parameters of power transformers. Along with the traditional criteria for insulation evaluating (resistance and the tangent of angle of dielectric loss of insulation, breakdown voltage of oil, etc.) which have a long history of practical experience, additional indicators 


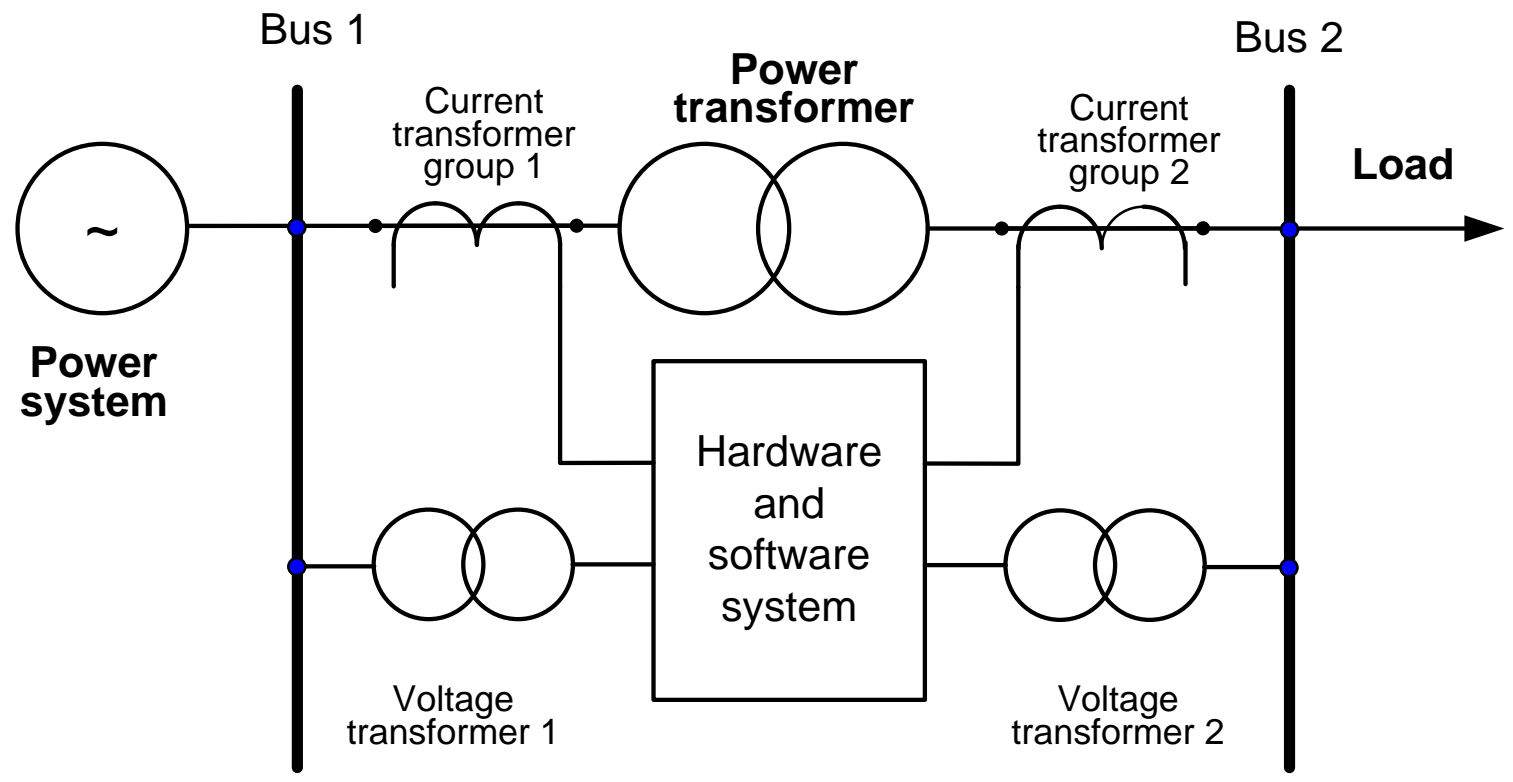

Fig. 1. Connection diagram of hardware and software complex for power transformer diagnostics.

are introduced. These include: chromatographic analysis of gases dissolved in oil, measurements of polymerization degree, control of furan content in oil, measurements of short circuit resistance, thermal imaging control.

Experience shows that although traditional tests are necessary and are the basis for assessment of transformer condition, they do not always enable to detect defects at an early stage and provide timely information on the development of processes that lead to a decrease in reliability and performance of equipment. Therefore, the use of additional controlled parameters is objectively justified and is developing both in Russia and other countries. At the same time, the system of regulatory parameters for assessing the condition of power transformers in operation is also being improved [1, 10$12]$.

Unfortunately it is impossible to accurately predict the degree of wear of transformer windings insulation by analyzing the impact of operational factors. This is explained by a rather complicated network of chemical reactions in insulation, leading to degradation of cellulose, and a vast amount of reasons that affect their development.

The aim of this study is to increase the accuracy of assessing the condition of transformer according to parameters of its normal operation mode. It is implemented by monitoring a number of the most important diagnostic indicators of transformer without disconnecting it from the network. This can be achieved by collecting and monitoring additional parameters and generating a warning signal in case when the monitored parameter exceeds the permissible values.

\section{2 materials and methods}

To achieve this goal it is necessary to solve a number of tasks. In this framework, the magnitudes and phase angles of voltages and currents of all transformer windings are measured in two different load conditions according to the circuit shown in the figure 1 .

The measured values are used to determine the resistance and short circuit loss, current and idle loss by solving the equations of the power transformer state, compiled on the basis of equivalent circuit of transformer. The technical condition and operability of transformer are assessed judging from excess of the obtained values over the corresponding reference values. By choosing transformer operation modes with different loads, the measured currents of windings and voltage drops are recorded. Further the first harmonic component of the registered currents and voltages of windings is derived, their symmetric components are determined, which are used in subsequent calculations. The zero phase-sequence resistance is determined by the parameters of asymmetric mode. This value is compared with the reference value, and the technical condition of electrical circuits connecting the transformer windings to the ground is determined by deviation of this value from the reference one. The active and inductive resistances of each of windings are determined and are used to assess the technical state of winding insulation and the state of current-carrying parts of windings. The transformer transformation coefficient is determined and is used to specify the technical condition of transformer voltage regulation device.

An improved method for determining the parameters of direct and zero sequence of power transformers will differ from the existing ones in that it will enable 
Table 1. Characteristics of the hardware and software complex.

\begin{tabular}{|l|l|l|}
\hline № & \multicolumn{1}{|c|}{ Parameter } & \multicolumn{1}{c|}{ Value } \\
\hline 1 & Number of measuring channels & Not less than 16 \\
\hline 2 & Accuracy class & $0.2 \mathrm{~S}$ \\
\hline 3 & Sampling frequency & $250 \mathrm{kHz}$ \\
\hline 4 & Transformer voltage & $6-110 \mathrm{kV}$ \\
\hline 5 & Supply voltage & $80-240 \mathrm{~V}$ \\
6 & Operation temperature & $-40-+65^{\circ} \mathrm{C}$ \\
& Interfaces & RS485, USB, Ethernet, $4-20 \mathrm{~mA}$ \\
\hline 7 & Power consumption, W & Determined by the research results \\
\hline 8 & Mass, g & Determined by the research results \\
\hline 9 & Dimensions LxWxH, mm & Determined by the research results \\
\hline
\end{tabular}

determining the parameters of a multi-winding (including two- and three-winding) transformer for an arbitrary position of regulator under load, different groups and connection schemes of windings based on data from digital registration of processes.

The algorithm for proactive control of the degree of deformation of windings of power transformers based on data of registration of emergency processes is also different in that the development of a defect can be established in the operating mode and thereby prevents occurrence of a sudden accident.

Early detection of potential damage will allow one to gain a sufficient margin of time to localize the damage without using an emergency shutdown.

The hardware and software complex for diagnosing internal damage in a power transformer is proposed to connect to measurement cores of current and voltage transformers with accuracy class 0.2 or 0.5 . The changes in equivalent circuit parameters should be monitored in normal conditions. This is sufficient to predict the development of winding faults and assess the windings deformation. The characteristics of the hardware and software complex are given in the table 1 .

\section{Results and discussions}

The economic effect of the study will be in reduction of damage from technological failures caused by sudden damage to the transformers, and, therefore, in reduction of risk of sudden disturbances in power system and interruptions in power supply to consumers.

We estimate the approximate damage from an emergency shutdown of a transformer with a rated power of $\mathrm{S}_{\mathrm{B}}=63 \mathrm{MV} \cdot \mathrm{A}$ due to damage to its windings.

Complete economic losses (disbenefit) arising from damage to the power transformer include damage from equipment damage and damage from interruptions in power supply to consumers:

$$
\mathrm{D}=\mathrm{D}_{\mathrm{EQ}}+\mathrm{D}_{\mathrm{US}}, \$
$$

where $D_{\mathrm{EQ}}$ is the losses from equipment damage, \$.

$D_{\mathrm{US}}$ is the losses from interruptions in power supply to consumers, $\$$.
We estimate the approximate damage from an emergency shutdown of transformer with rated power $S_{\mathrm{B}}=63 \mathrm{MV} \cdot \mathrm{A}$ due to damage to its windings.

The average specific damage per unit transformer power, according to [7], is $d=8990 \$ / \mathrm{MV} \cdot \mathrm{A}$.

According to data of Table 4 [7] the damage rate of transformer windings $k$ of the total number of damage is:

$$
\mathrm{k}=\frac{\mathrm{N}}{\mathrm{N}_{\Sigma}} \cdot 100 \%,
$$

where $N$ is the amount of internal damage to transformers;

$N_{\Sigma}$ is the total damage.

$$
\mathrm{k}=\frac{24}{94} \cdot 100 \%=25.5 \% \text {. }
$$

Then the expected losses from the emergency shutdown of transformer with a rated power of $\mathrm{S}_{\mathrm{B}}=63$ $\mathrm{MV} \cdot \mathrm{A}$ due to damage to its windings will be:

$$
\mathrm{D}=\mathrm{k} \cdot \mathrm{d} \cdot \mathrm{S}_{\mathrm{B}}=0.255 \cdot 8990 \cdot 63=144420 \$ \text {. }
$$

Analysis of the final data of Table 1 [7] shows that the share of losses caused by equipment damage is $56.95 \%$, and the share of losses from interruptions in power supply accounts for $43.05 \%$.

Taking into account formula (1), we obtain:

$$
\begin{aligned}
& D_{\mathrm{EQ}}=82250 \$ ; \\
& D_{\mathrm{US}}=62170 \$
\end{aligned}
$$

According to $[1,8,9]$, the damageability of new transformers is $0.2 \%$ (every 500th transformer). For the 30 -years transformers this value is $1 \%$ (every 100th transformer). According to [10], for transformers and autotransformers with voltage of $110-500 \mathrm{kV}$ and power of $63 \mathrm{MV} \cdot \mathrm{A}$ or more, operating in electric networks, the specific damage, accompanied by the occurrence of internal short circuits, is $0.45 \%$ per year or every $222 \mathrm{nd}$ transformer. In this case, damage to the windings occurs in transformers with any service life [1].

Installing the proposed hardware and software complex on 222 transformers will prevent the annual 
expected losses valued at $\$ 144,420$. Analysis of market offers of leading manufacturers of microprocessor devices showed that the estimated cost of one set of hardware and software kit will be $\$ 1270$, so the investment in 222 transformers should be $\$ 281,940$.

\section{Conclusions}

The presented results promote opportunities of analysis the condition of transformers in operating mode. They can be used in construction of various monitoring systems to identify developing transformer defects caused by windings damage. The implementation of algorithm for monitoring the degree of windings deformation in the protection against internal damage allows one to prevent the approaching moment of damage and prevent occurrence of sudden accidents.

The proposed diagnostic system enables comprehensive assessment of power transformers condition, make decisions on their further operation and implement the technical policy of transition to repair according to the actual equipment condition. Calculations show that the discounted payback period for capital investments in this system will be about two years.

\section{References}

[1] B.V. Anin, Yu.N. L’vov, M.Yu. L’vov, B.N. Neklepaev, K.M. Antipov, A.S. Surba, M.I. Chitinsky, Power stations 9 (2001).

[2] S. Miyazaki, Yo. Yamamasu, H. Sakaki, Method and device for diagnosing soundness in transformer, Patent JP2013061310A, publication of 2013-04-04.

[3] Z. Hui-Min, Three-phase three-winding transformer fault area diagnostic method Publication, Patent CN104076227A, Publication of 2014-10-01.

[4] M. Mostafaei, J. Faiz, P. Venikar, M. Sudhakar, Ballal Turn-to-turn fault monitoring methods in electrical power transformers-State of the art, International Transactions on Electrical Energy Systems 28, 2 (2018). DOI: 10.1002/etep.2644.

[5] E. Kornatowski, S. Banaszak, IEEE Transactions 29, 1398-1406 (2014). DOI: 10.1109/TPWRD.2013.2289944.

[6] F. Haghjoo, M. Mostafaei, H. Mohammadi, IEEE Transactions on Power Delivery, 1-1 (2017). DOI: 10.1109/TPWRD.2017.2688419.

[7] W. Bartley, Power Engineering and Management 1, 58 (2011).

[8] B.V. Vanin, Yu.N. Lvov, M.Yu. Lvov, et al., Damage to $110-500 \mathrm{kV}$ power transformers in operation, Power stations 9 (2001).

[9] V.S. Bogomolov, N.N. Khublarov, M.Yu. A.O. Lvov, Problems of autotransformers operation in systems with fault currents upgrowth, CIGRE 12 (2000).

[10] M.Yu. Lvov, Power transformers of $110 \mathrm{kV}$ and higher, Diagnostics will determine the future, Electrical News 6, 24 (2003).
[11] A.N Alyunov, O.S. Vyatkina, L.R. Mukhametova, V.P. Markovskiy, R.M. Mustafina, E3S Web of Conferences 124, 02016 (2019). DOI: 10.1051/e3sconf/201912402016.

[12] A.E. Nemirovskiy, A.I. Kashin, V.F. Kosmach, Yu.F. Titovec, I.N. Toptygin, D.A. Zaripova, Innovative technology for dismantling the windings of electric motors using ultrasonic radiation, IOP Conference Series: Earth and Environmental Science 337 (2019). DOI: 10.1088/1755-1315/337/1/012071. 